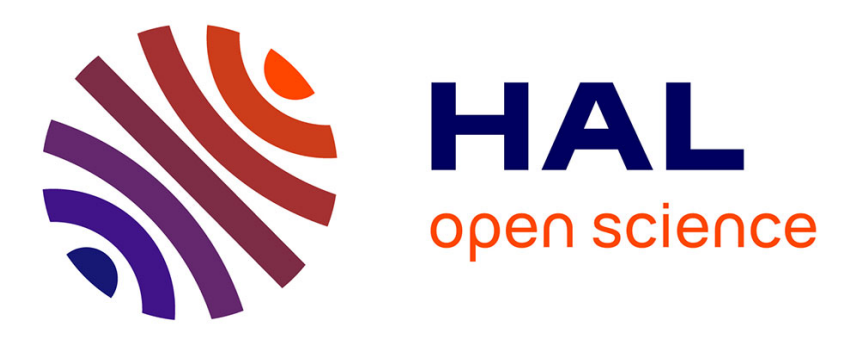

\title{
Extrinsic calibration between a multi-layer lidar and a camera
}

\author{
Sergio Alberto Rodriguez Florez, Vincent Fremont, Philippe Bonnifait
}

\section{To cite this version:}

Sergio Alberto Rodriguez Florez, Vincent Fremont, Philippe Bonnifait. Extrinsic calibration between a multi-layer lidar and a camera. IEEE International Conference on Multisensor Fusion and Integration for Intelligent Systems, MFI 2008, Aug 2008, South Korea. pp.214-219, 10.1109/MFI.2008.4648067 . hal-00444112

\section{HAL Id: hal-00444112 \\ https://hal.science/hal-00444112}

Submitted on 5 Jan 2010

HAL is a multi-disciplinary open access archive for the deposit and dissemination of scientific research documents, whether they are published or not. The documents may come from teaching and research institutions in France or abroad, or from public or private research centers.
L'archive ouverte pluridisciplinaire HAL, est destinée au dépôt et à la diffusion de documents scientifiques de niveau recherche, publiés ou non, émanant des établissements d'enseignement et de recherche français ou étrangers, des laboratoires publics ou privés. 


\title{
Extrinsic Calibration between a Multi-Layer Lidar and a Camera
}

\author{
Sergio A. Rodriguez F., Vincent Frémont and Philippe Bonnifait
}

\begin{abstract}
In this paper, we present a novel approach for solving the extrinsic calibration between a camera and a multi-layer laser range finder. Our approach is oriented for intelligent vehicle applications, where the separation distance between sensors frames are frequently very important. For this purpose, we use a circle-based calibration object because its geometry allows us to obtain not only an accurate estimation pose by taking advantage of the 3D multi-layer laser range finder perception but also a simultaneous estimation of the pose in the camera frame and the camera intrinsic parameters. These advantages simplify the calibration task in outdoor environments. The method determines the relative position of the sensors by estimating sets of corresponded features and by solving the classical absolute orientation problem. The proposed method is evaluated by using different synthetics environments and real data. An error propagation analysis is made in order to estimate the calibration accuracy and the confidence intervals. Finally, we present a laser data projection into images to validate the consistency of the results.
\end{abstract}

\section{INTRODUCTION}

In the framework of intelligent vehicles, multi-sensor systems are used for developing complete preventive architectures in order to improve vulnerable road user protection systems. These multi-sensor systems are usually composed with radars, laser range-finders and cameras. In many applications like obstacle and pedestrian detection and tracking systems, it is necessary to fuse information supplied by each sensor in a cooperative way to associate the advantages of every individual system in a final cooperative structure. For this purpose the relative pose of the sensors have to be estimated. Previous works on extrinsic laser-camera calibration solve with accuracy the rigid transformation between a CCD camera and a one-row laser range-finder [1] [2]. Another calibration approach for outdoor scan systems without using a calibration target is presented in [3]. Estimating the geometrical transformation in one thing, but it is often important to know the quality of the calibration between the frames of different sensors for estimating guaranteed regions of interest (ROI). Therefore, a confidence information on the calibration stage is of prime importance.

In this paper, we propose an extrinsic calibration method, vehicle-oriented, for a particular relative position between a camera and a multi-layer laser lidar. It is worth to mention that our contribution is focused on the calibration of the multi-sensor system but not on each sensor. This particular configuration is characterized by two critical conditions.

Manuscript received February 22, 2008. Sergio A. Rodriguez F., Vincent Frémont and Phillipe Bonnifait are with Heudiasyc UMR CNRS 6599, Université de Technologie de Compiègne, BP20529 - 60205 Compiègne Cedex, France. Emails: srodriguahds.utc.fr, vincent. fremontahds.utc. fr and philippe.bonnifaitautc.fr
The first critical point consists in the necessity to have an important relative distance between the sensors and the calibration target (see Figure 1). The increase of the relative distance between the camera and the calibration target demands a large-size calibration target in order to ensure the accuracy of the estimation pose in the camera frame. A second point is the large noise detected on 3D laser range finder measurements detecting the surface of the traditional "checkerboard" calibration object. This increase is partially caused by the laser beam impacts on blackwhite zones. Hence, our principal objectives are focused on getting an accurate rigid transformation, a size-reduction of the calibration target, a method which takes advantage of the 4-layer lidar information and an error analysis and confidence interval estimation.

This paper is outlined as follows. Section II presents the extrinsic calibration problem, the mathematical multi-sensor system model, theorical basis and definitions. In section III, we present the proposed calibration method summarized in a circle-based target pose estimation step, rigid transformation computation step and error/confidence analysis. Then, the calibration algorithm summary is clarified in section IV. Finally, results obtained with simulated data and real acquisitions are presented in section $\mathrm{V}$.

\section{MULTI-SENSOR SYSTEM}

The multi-sensor system is made with a multi-layer range finder and a CCD camera rigidly fixed to the vehicle. The multi-layer lidar is located in the bumper section and the camera is placed behind the windshield (see Figure1). This positioning only presents some occlusions to the camera for short distances with respect to the multi-layer range finder.

\section{A. Problem Statement}

The problem consists in calculating the rigid transformation (6 dof corresponding to a rotation matrix and a translation vector) between the camera and the 4-layer lidar frames. In order to obtain an accurate estimation of the extrinsic parameters, we propose a method which takes advantage of photographic and laser range data by using a circle-based calibration target. It is worth to mention that the laser beam of the lidar sensor is invisible (see Figure 1). The circle-based calibration target is a rigid plane with a printed black ring. The inner circle of the black ring describes a plane perforation. Some of the benefits of using the proposed calibration target are the following: Firstly, the circle based target eliminates the noise generated by the black-white zones of the traditional "checkerboard" calibration target. Secondly, it is possible to obtain a precise estimation of the 
pose by computing a circle fitting in the 3D space of the lidar impacts lying in the perforation border of the calibration target. Thirdly, the geometric and algebraic constraints of the two concentric circles generated by the black ring, and their image projection allow us to obtain a simultaneous estimation of the camera pose and the intrinsic parameters (i.e. focal length).

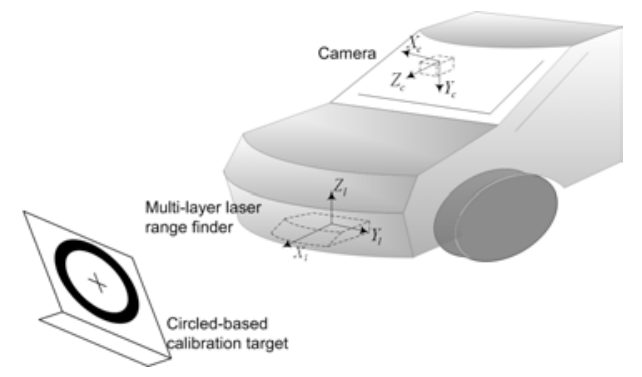

Fig. 1. Overview of the proposed strategy

\section{B. Sensor Models and Frames}

Our multi-layer lidar model uses 4 crossed-scan-planes with a layer relative altitude aperture of $0.8^{\circ}$ and an azimuthal angle resolution defined as follows: $0.125^{\circ}$ in the range $\left[-16^{\circ} 16^{\circ}\right], 0.25^{\circ}$ for $\left[-60,-16^{\circ}\right)$ and $\left(16^{\circ}, 60\right]$ and $0.5^{\circ}$ for $\left[-70,-60^{\circ}\right)$ and $\left(60^{\circ}, 70\right]$. Figure 2 illustrates in a general way the emission direction of the laser layers. Let be $i$ the layer id and $j$ the scan point element. Thus, a 3D laser impact is defined in the lidar frame, $l$, as: $\left.{ }^{l} \mathrm{P}_{i j}=\left[{ }^{l} P_{x},{ }^{l} P_{y},{ }^{l} P_{z}\right]\right]^{T}$

For the purpose to define any lidar point with respect to the calibration object frame (i.e. target frame, $t$ ), it is necessary to define a rigid transformation. Therefore, the corresponded point of ${ }^{l} \mathrm{P}_{i j}$ in the target frame, noted ${ }^{t} \mathrm{P}_{i j}$, can be obtained by applying the following rigid transformation:

$$
{ }^{t} \mathrm{P}_{i j}={ }^{t} \mathbf{R}_{l} \cdot{ }^{l} \mathrm{P}_{i j}+{ }^{t} \mathrm{~T}_{l}
$$

It is worth adding that the calibration plane is defined by $Z_{t}=0$ and the origin of the target frame is physically placed at the center of target circle. Similarly as in equation (1), a point in the target frame, ${ }^{t} \mathrm{P}$, is defined in the camera frame, $c$, as follows:

$$
{ }^{c} \mathrm{P}_{i j}={ }^{c} \mathbf{R}_{t} \cdot{ }^{t} \mathrm{P}_{i j}+{ }^{c} \mathrm{~T}_{t}
$$

with ${ }^{c} \mathrm{P}_{i j}=\left[\begin{array}{lll}{ }^{c} P_{x}, & { }^{c} P_{y}, & { }^{c} P_{z}\end{array}\right]^{T}$

Where ${ }^{c} \mathbf{R}_{t}$ is a $3 \times 3$ orthonormal matrix representing the orientation and ${ }^{c} T_{t}$ a 3 -vector representing the position of the calibration target in the camera frame.

Let us consider the complete multi-sensor model where the data provided by the lidar and the camera are related by using a common detected object (i.e. circle-based calibration target). We proceed to formalize the lidar to camera transformation as a composition of the partial transformations presented in equations (1) and (2).
Therefore, the rigid transformation of a $3 \mathrm{D}$ point in the lidar frame, ${ }^{l} \mathrm{P}_{i j}$, into the camera frame is determinated by replacing equation (1) into (2) obtaning:

$$
{ }^{c} \mathrm{P}_{i j}={ }^{c} \mathbf{R}_{t} \cdot\left({ }^{t} \mathbf{R}_{l} \cdot{ }^{l} \mathrm{P}_{i j}+{ }^{t} \mathrm{~T}_{l}\right)+{ }^{c} \mathrm{~T}_{t}
$$

simplyfing,

$$
{ }^{c} \mathrm{P}_{i j}=\boldsymbol{\Phi} \cdot{ }^{l} \mathrm{P}_{i j}+\Delta
$$

with $\boldsymbol{\Phi}={ }^{c} \mathbf{R}_{t} \cdot{ }^{t} \mathbf{R}_{l}$ and $\Delta={ }^{c} \mathbf{R}_{t} \cdot{ }^{t} \mathrm{~T}_{l}+{ }^{c} \mathrm{~T}_{t}$ respectively the orientation and the position of the lidar sensor with respect to the camera. $[\boldsymbol{\Phi}, \Delta]$ are the unknown values of the calibration problem.

Additionally, the impact location of the lidar measurements can be projected into the image even if the lidar beam is invisible. For this purpose, a classical pinhole model is considered. Hence, the image projection of a 3D point in the camera frame, ${ }^{c} \mathrm{P}_{i j}$, is given by

$$
\left[p_{x}, p_{y}, 1\right]^{T} \sim \mathbf{K} \cdot{ }^{c} \mathrm{P}_{i j}
$$

with

$$
\mathbf{K}=\left(\begin{array}{ccc}
f & 0 & u_{0} \\
0 & f & v_{0} \\
0 & 0 & 1
\end{array}\right)
$$

where $\mathbf{K}$ is the intrinsic calibration matrix with $f$ the focal length of the camera in pixels units and $\left[u_{0}, v_{0}\right]^{T}$ the image coordinates of the principal point, assuming no distortion and zero skew. The principal point is considered as a known parameter. The operator $\sim$ denotes : up to a scale factor. Finally, the image projection of ${ }^{l} \mathrm{P}_{i j}$ is computed by using equation (4) in (5):

$$
\left[p_{x}, p_{y}, 1\right]^{T} \sim \mathbf{K} \cdot\left(\boldsymbol{\Phi} \cdot{ }^{l} \mathrm{P}_{i j}+\Delta\right)
$$

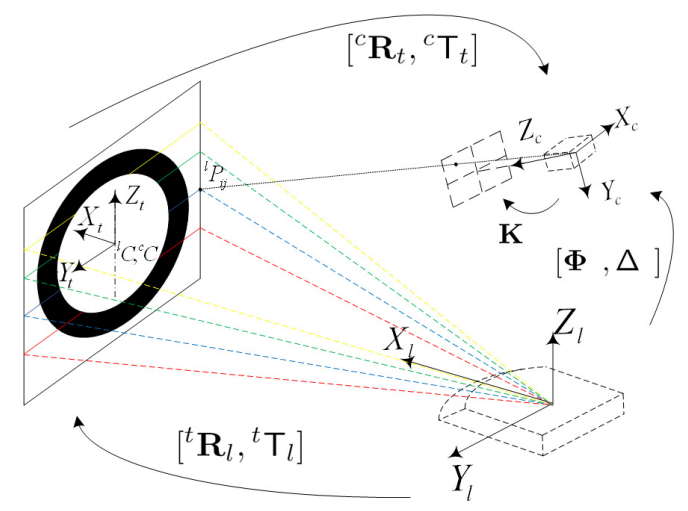

Fig. 2. Rigid transformations

\section{EXTRINSIC CALIBRATION}

The proposed solution consists in estimating different poses of the calibration object detected simultaneously by the camera and the multi-layer lidar. A minimum of 6 poses [4] have to be estimated in the lidar and the camera frame in order to get all degrees of freedom. Each pose of the calibration target is parameterized by the $3 \mathrm{D}$ coordinates of the circle center and the normal vector of its plane. Then, 
a first estimation of the rigid transformation is obtained by solving the usual absolute orientation problem [4]. This solution consists in determining the relationship between the two coordinate frames using sets of corresponded features (i.e. circle centers of each pose). Finally a non-linear 3D minimization is done in order to refine the estimated extrinsic parameters.

\section{A. Target pose estimation in the lidar frame}

First, for a robust detection of the circle in the 3D space, we use several lidar scans. Then, the robust outlier rejection technique proposed in [1] is applied on $n$ 4-layer lidar scans of the calibration scene. After the outlier rejection proceeding, the points lying in the perforation border of the calibration target are extracted. This point set contains between 8 and $8 \cdot n$ points denoted ${ }^{l} \mathrm{P}_{i}$. It is worth to take into account that the pose of target frame origin with respect to the lidar frame is defined as by the inversed transformation stated in equation (1) where ${ }^{t} \mathbf{R}_{l}^{T}$ corresponds to the orientation of the calibration frame and its origin in the lidar frame, ${ }^{l} \mathrm{C}=-\left({ }^{t} \mathbf{R}_{l}^{T} \cdot{ }^{t} \mathrm{~T}_{l}\right)$. Taking advantage of this fact, we perform a nonlinear $3 \mathrm{D}$ circle fitting problem constrained to a known radius, $r$, and parameterized as follows: $\hat{\alpha}, \hat{\beta}$ are orientation angles of the $3 \mathrm{D}$ circle axis vector, ${ }^{l} \mathrm{~N}(\hat{\alpha}, \hat{\beta})$, with respect to y-axis and z-axis respectively and ${ }^{l} \hat{\mathrm{C}}=\left[{ }^{l} \hat{C}_{x},{ }^{l} \hat{C}_{y},{ }^{l} \hat{C}_{z}\right]^{T}$ are the cartesian coordinates of the estimated 3D circle center coincident with the target frame origin (see Figure 2). By using the geometric criteria as stated in [5]:

$$
\begin{aligned}
& { }_{i} \Pi_{1}={ }^{l} \mathrm{~N}(\hat{\alpha}, \hat{\beta}) \cdot \overrightarrow{{ }^{l} \hat{\mathrm{C}}^{l} \mathrm{P}_{i}} \\
& { }_{i} \Pi_{2}=\left\|{ }^{l} \mathrm{~N}(\hat{\alpha}, \hat{\beta}) \times{ }^{l} \hat{\mathrm{C}}^{l} \mathrm{P}_{i}\right\|-r
\end{aligned}
$$

with $\overrightarrow{{ }^{l} \hat{\mathrm{C}}^{l} \mathrm{P}_{i}}={ }^{l} \mathrm{P}_{i}-{ }^{l} \hat{\mathrm{C}}$.

Where:

- ${ }_{i} \Pi_{1}$ corresponds to the Euclidean distance between a target-contour laser range finder impact, ${ }^{l} \mathrm{P}_{i}$, and the 3D plane defined by ${ }^{l} \mathrm{~N}(\hat{\alpha}, \hat{\beta})$ and the estimated circle center, ${ }^{l} \hat{\mathrm{C}}$.

- ${ }_{i} \Pi_{2}$ represents the Euclidean distance between a target-contour laser range finder point, ${ }^{l} \mathrm{P}_{i}$, and the $3 \mathrm{D}$ circle axis defined by ${ }^{l} \mathrm{~N}(\hat{\alpha}, \hat{\beta})$ and the estimated circle center, ${ }^{l} \hat{\mathrm{C}}$.

Accordingly, we minimize the following objective function using the Levenberg-Marquardt algorithm (LM-algorithm) [6] which solves the following non-linear squares problem:

$$
e=\sum_{i=1}^{n}\left[{ }_{i} \Pi_{1}^{2}+{ }_{i} \Pi_{2}^{2}\right]
$$

After convergence of the non linear minimization algorithm and by applying this technique to various poses ( 6 poses are needed for a solution) of the calibration target, we obtain not only a first set of $3 \mathrm{D}$ laser features (i.e. circle centers, ${ }^{l} \hat{C}$, and normal plane vectors, ${ }^{l} \mathrm{~N}$ ) but also a $3 \mathrm{D}$ circle reconstruction in the laser range finder frame for every pose. Now, in order to acquire the corresponded features in the camera frame we have to analyze the image.

\section{B. Target pose estimation in the camera frame}

Like several camera calibration methods using projected concentric circles [7] [8] [9], we estimate the position of the calibration target in the camera frame. For this purpose, it is necessary to estimate the intrinsic camera parameters. As stated in [7], intrinsic calibration parameters can be obtained first by computing the image of the absolute conic (IAC) with precision [10] from the imaged circular points [11] [10] with only an image of two concentric circles. Considering the intrinsic calibration issue as a non principal aim of this paper, we will not give more details about it. Given $A_{1}$ and $A_{2}$ as the pixel centered point set of the principal (i.e. external) and secondary (i.e. internal) circle projection which can be obtained by the segmentation methods widely explained in [9] [12]. Achieving a non linear ellipse fitting algorithm stated in [13], we obtain two conic matrices, $\mathbf{Q}_{1}$ and $\mathbf{Q}_{2}$, in the form:

$$
\mathbf{Q}=\left(\begin{array}{ccc}
\mathbf{a} & \mathbf{b} / 2 & \mathbf{d} / 2 f \\
\mathbf{b} / 2 & \mathbf{c} & \mathbf{e} / 2 f \\
\mathbf{d} / 2 f & \mathbf{e} / 2 f & \mathbf{f} / f^{2}
\end{array}\right)
$$

Where $\mathbf{a}, \mathbf{b}, \mathbf{c}, \mathbf{d}, \mathbf{e}, \mathbf{f}$ are the conic parameters and $f$ is the focal length in pixels. $\mathbf{Q}_{1}$ and $\mathbf{Q}_{2}$ are normalized to $\operatorname{det} \mathbf{Q}=$ -1 . Consequently, in order to remove the scale uncertainty, the normal vector to the target plane as in [8] is given by:

$$
{ }^{c} \mathbf{N}=\mathbf{Q}_{1}\left[\begin{array}{c}
x_{c} \\
y_{c} \\
f
\end{array}\right]
$$

Where $x_{c}$ and $y_{c}$ are the image coordinates of the projected circle center. Finally, the 3D circle center in the camera frame, ${ }^{c} \hat{\mathrm{C}}$, is obtained as stated in [9]:

$$
{ }^{c} \hat{\mathrm{C}}=\frac{\sqrt{\lambda^{3}} R\left[x_{c} / f y_{c} / f 1\right]^{T}}{{ }^{c} \mathrm{~N} \cdot\left[x_{c} / f y_{c} / f 1\right]^{T}}
$$

Where $\lambda$ is the smaller positive eigenvalue of $Q_{1}$ and $R$ is the radius of the principal circle in the target object. Thus, the camera pose estimation is characterized by ${ }^{c} \mathrm{~N}$ and ${ }^{c} \hat{\mathrm{C}}$.

\section{Estimation of the rigid transformation between the lidar and the camera}

The method presented in the above subsection allows us estimating the 3D center points of the circle-based calibration target for various poses. These pose estimations are composed with $3 \mathrm{D}$ corresponding center point set in the camera and the laser range finder frame. Therefore, in order to estimate an initial guess solution we formulate the extrinsic calibration as a classical absolute orientation problem.

1) Initial guess from a linear solution: A well-known closed-form solution for this problem is the method developed by Arun et al. [14]. This method consists in obtaining the optimal rotation from the singular value decomposition (SVD) of the correlation matrix of the centered point sets represented by $\Sigma$ :

$$
\boldsymbol{\Sigma}=\left[{ }^{l} \hat{\mathrm{C}}_{i}-{ }^{l} \overline{\mathrm{C}}\right]\left[{ }^{c} \hat{\mathrm{C}}_{i}-{ }^{c} \overline{\mathrm{C}}\right]^{T}=\mathbf{U} \mathbf{S} \mathbf{V}^{T}
$$

Where ${ }^{l} \hat{C}_{i}$ are the coordinates of the $3 \mathrm{D}$-circle center point set estimated from the $i^{t h}$ pose by the laser range finder 
measures, ${ }^{l} \overline{\mathrm{C}}$ is the centroid of the $3 \mathrm{D}$-circle center point set in the laser range finder frame, ${ }^{c} \hat{\mathrm{C}}_{i}$ are the coordinates of the 3D-circle center point set estimated from the $i^{t h}$ pose by the camera measures and ${ }^{c} \overline{\mathrm{C}}$ is the centroid of the $3 \mathrm{D}$-circle center point set in the camera frame. Thus, the $3 \times 3$ optimal rotation matrix is obtained as follows:

$$
\mathbf{\Phi}_{0}=\mathbf{V} \mathbf{U}^{T}
$$

The translation, $\Delta_{0}$, is obtained as the vector which aligns the centroid of the 3D-circle center point set in the camera frame, ${ }^{c} \overline{\mathrm{C}}$, and the rotated centroid $\boldsymbol{\Phi}_{0} \cdot{ }^{l} \overline{\mathrm{C}}$ :

$$
\Delta_{0}={ }^{c} \overline{\mathrm{C}}-\boldsymbol{\Phi}_{0} \cdot{ }^{l} \overline{\mathrm{C}}
$$

2) Refining parameters: The rigid transformation obtained in the above section, $\left[\boldsymbol{\Phi}_{0}, \Delta_{0}\right]$, is a linear minimization of the Euclidean distance error between the $3 \mathrm{D}$ circle center point sets. This solution is usually a good starting guess of the extrinsic calibration. Therefore, in the aim of refining these estimated parameters, we first generate the 3D circles of the $n$ poses estimated by the camera. It consists in computing $m$ points of each estimated circle pose by using the 3D circle center and an orthonormal base lying in circle's plane. This orthonormal base is obtained from the normal vector to the circle's plane applying the GramSchmidt procedure [15]. Let be ${ }^{c} \mathrm{~F}_{i, k}$, the $k^{\text {th }}$ generated 3D point using the camera estimation of the $i^{t h}$ pose. Secondly, the 3D circles of all the poses estimated by the lidar are generated in the same way as presented for the camera estimations obtaining ${ }^{l} \mathrm{~F}_{i, k}$. Then, we apply systematically the first guess for the rigid transformation, $\left[\boldsymbol{\Phi}_{0}, \Delta_{0}\right]$, as in the equation (4). Thirdly, under the assumption that the error orientation of the first guess rigid transformation is lower than $\pi / 2$, we match the 3D points of the camera and lidar transformed estimations for every pose by using the nearest neighbor criterion as illustrated in Figure 3. At this point, it is worth to mention that we have a $3 \mathrm{D}$ point set of camera and lidar observations associated. Finally, the refining of the rigid transformation parameters, $\left[\alpha, \beta, \rho, t_{x}, t_{y}, t_{z}\right]^{T}$, is obtained by minimizing the following non-linear objective function:

$$
\epsilon=\sum_{i=1}^{n} \sum_{k=1}^{m} \mathbf{W} \cdot\left[D_{i k}^{2}\right]
$$

with $D_{i k}=\left\|{ }^{c} \mathrm{~F}_{i, k}-\left[\boldsymbol{\Phi}_{(\alpha, \beta, \rho)} \cdot{ }^{l} \mathrm{~F}_{i, k}+\Delta_{\left(t_{x}, t_{y}, t_{z}\right)}\right]\right\|$.

Where $D_{i k}$ represents the Euclidean distance residual of the points after applying the rigid transformation and $\mathbf{W}$ is a weighting matrix. The results are obtained by using a robust M-estimator algorithm for calculating the robust weights as stated in [16] and the LM-algorithm. After convergence, the solution of the calibration problem is represented by $[\boldsymbol{\Phi}, \Delta]$.

\section{Calibration accuracy}

At this point, we have estimated the rigid transformation between the camera and the laser range finder frame. The accuracy of the calibration results is estimated under the assumption that measurement errors are normally distributed.

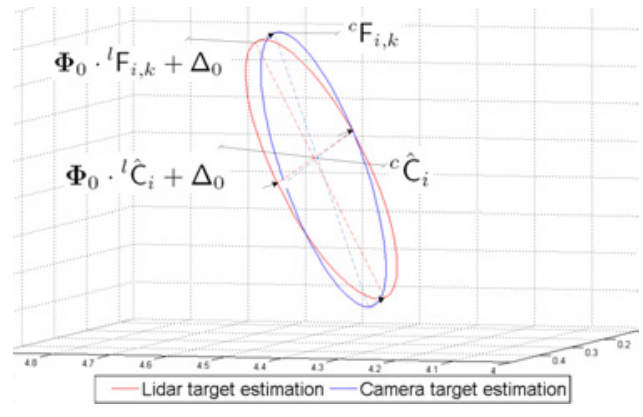

Fig. 3. Matching of the camera and lidar estimations

Therefore, the covariance matrix of the estimated parameters, $\mathbf{C}_{\sigma}$, is defined as follows:

$$
\mathbf{C}_{\sigma}=M S E \cdot \mathbf{J} \cdot \mathbf{J}^{T}
$$

with $M S E=\frac{1}{\theta-\phi} \cdot \sum_{i=1}^{\theta} \epsilon^{2}$

Where $\mathbf{J}$ represents the Jacobian matrix of the last LMalgorithm iteration and MSE represents the mean squared error defined by $\theta$, the number of observations, $\phi$, the number of estimated parameters and $\epsilon$ the residual of the non-linear objective function. In our case, $\phi$ is equal to 6 (3 rotations and 3 translations) and $\theta-\phi$ represents the degree of freedom of the $\chi^{2}$ distribution. Based in the above classical approach for the covariance matrix of the non-linear fitted parameters, the width of the $95 \%$ confidence interval is obtained thanks to [17]:

$$
\delta \mathbf{C}_{i}=\sqrt{M_{\chi^{2}(95 \%, \phi)}} \cdot \sqrt{\mathbf{C}_{\sigma}(i, i)}
$$

where $\sqrt{\mathbf{C}_{\sigma}(i, i)}$ is the standard deviation of the estimated parameter and $M$ the corresponding value of the $\chi^{2}$ distribution function.

\section{CALIBRATION ALGORITHM}

The following is a summary of the calibration method explained in section III.

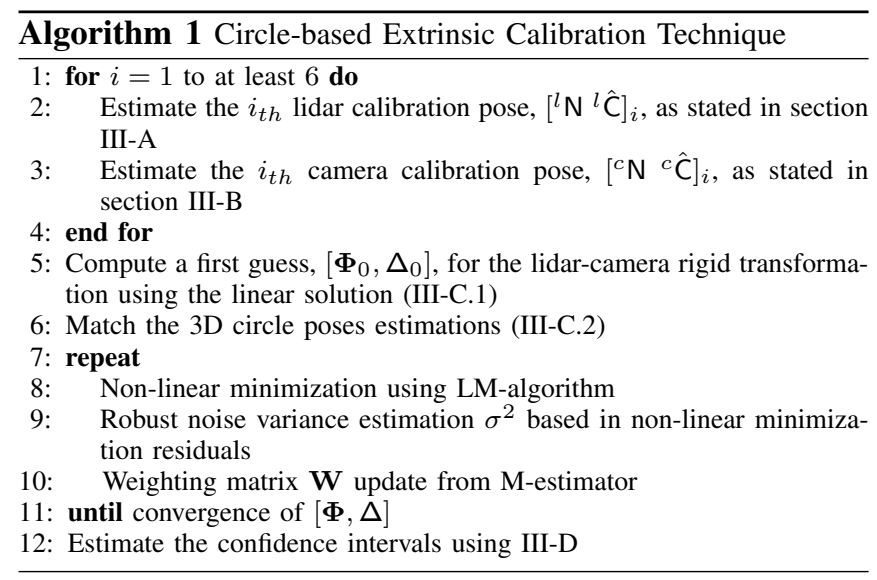

\section{EXPERIMENTAL RESULTS}

Evaluation tests have been carried out in order to estimate the behavior and robustness of the presented method in simulated and real conditions. The method has been implemented using Matlab 7.4. 


\section{A. Synthetic data}

The considerations which were taken into account in the simulation model correspond to the sensor relative position on board the vehicle. The extrinsic parameters used were the translation vector in meters $\Delta=[-0.2,0.8,1.8]^{T}$ and the orientation matrix $\boldsymbol{\Phi}_{\alpha, \beta, \rho}$, computed from the rotation angles $\alpha=11^{\circ}, \beta=-1^{\circ}$ and $\rho=0.5^{\circ}$. The multi-layer lidar impacts were generated as the intersection of the lidar beam emission vectors and the simulated calibration target plane. A 3D space constraint was used to guarantee that all lidar layers impact the calibration object. Then, a Gaussian white noise was added in the direction of the lidar beam emission vector. By using the model presented in (5), a synthetic image was computed as a discrete image projection of the circlebased calibration target. The intrinsic parameters used in the camera model were a focal length of 1670 pixels and point $(0,0)$. Next, a Gaussian white noise was added to the image projection coordinates of the circle-based calibration target and to the focal length of the camera model.

1) Test No. 1: A first Monte Carlo-like simulation test was made in order to estimate the precision achieved by the method with a minimal number of poses (worst case). Thus, 6 random poses were distributed and oriented randomly in the common FOV of the multi-sensor system by 100 trials. The noise was added to the image coordinates and the focal length was fixed to a standard deviation of one pixel. For each trial the extrinsic parameters were estimated. The results obtained are presented in table I. The precision of the calibration method increase proportionally to the number of poses.

\begin{tabular}{|l|c|}
\hline \multicolumn{2}{|c|}{ Results of the Test No. 1 (Using only 6 poses) } \\
\hline Relative Position error (millimeters) & 46.1059 \\
\hline Relative Orientation error (degrees) & 3.4362 \\
\hline Iterations & 401 \\
\hline
\end{tabular}

TABLE I

PRECISION ACHIEVED UNDER A MINIMAL NUMBER OF POSES

2) Test No. 2: For evaluating the behavior of the method with regard to a noise increase, a second simulation test was performed. In the same way as the first simulation test, 100 trials using 7 poses were generated for each level of the Gaussian white noise added in a range between 1 to 3 pixels of standard deviation. In order to compare the results obtained by the robust non-linear minimization of the 3D poses (see III-C ) we have also executed an iterative closest point algorithm (ICP) [18] as a reference of a classical registration of 3-D point sets. Results are illustrated in Figure 4(a) and Figure 4(b). By the same, in Figure 4(c) the translation error behavior was estimated by changing the number of poses at a Gaussian white noise of 1 pixel of standard deviation.

3) Test No. 3: Thirdly, we present the results obtained in a test of consistency computed from the estimations of the rigid transformations and the confidence intervals using 7 poses in the calibration process. The Figure 5 illustrates as an example, the evolution of a rotation parameter over the $y$-axis in the lidar frame, $\beta$. The robust non linear algorithm improves the results and it has a good statistical efficiency

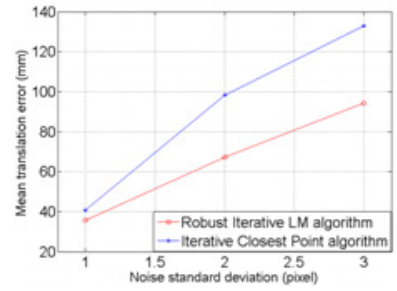

(a) Translational error (mm.)

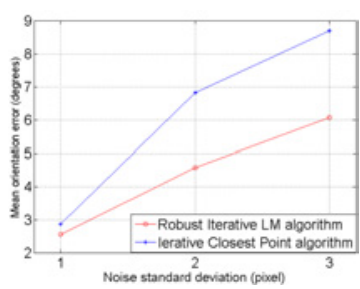

(b) Orientation error (degrees)

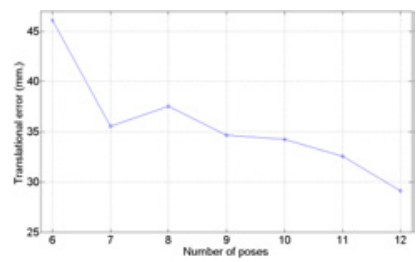

(c) Error behavior by poses

Fig. 4. Relative errors

but its convergence is not guaranteed as observed at the $53^{\text {th }}$, $69^{\text {th }}$ and $88^{\text {th }}$ trials.

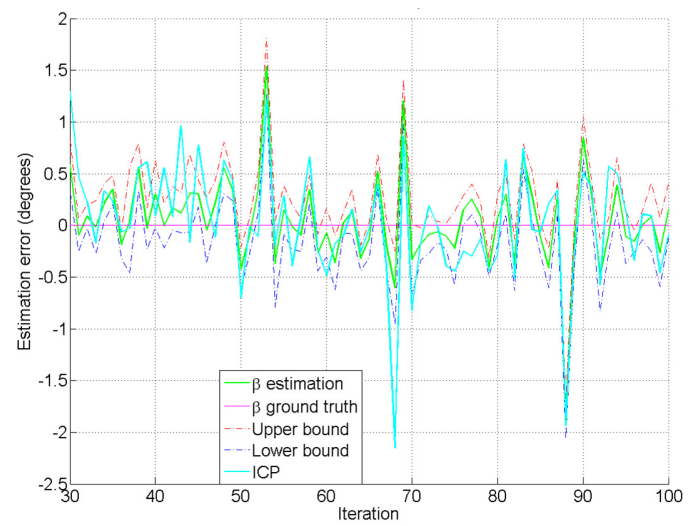

Fig. 5. Consistency test for $\beta$ at $2 \sigma$

\section{B. Real data}

Experiments using real data were made thanks to the experimental platform of the Heudiasyc laboratory (see Figure 6(a)). This vehicle is equipped with an IBEO Alasca XT and a camera Sony DFW-VL500. The resolution of the camera was set to $640 \times 480$ pixels. We have used a calibration target with 2 concentric circles of radii 25 $\mathrm{cm}$. and $20 \mathrm{~cm}$. A number of 20 scans were taken into account for each pose in the calibration process. Only 8 poses were used to estimate the initial guess solution for the rigid transformation. The real tests highlight some imprecisions of the target pose estimation in the camera frame, ${ }^{c} \mathrm{~N}_{i}$ and ${ }^{c} \hat{C}_{i}$. These imprecisions were observed in presence of high image noise levels. This problem was solved by minimizing the ellipse parameters error between the target image and the 2-D projection of the 3-D target estimation of the camera, which optimizes ${ }^{c} \mathrm{~N}_{i}$ and $\left\|{ }^{c} \hat{\mathrm{C}}_{i}\right\|$. After the convergence of the non-linear minimization algorithm (see section III-C.2) the computed extrinsic parameters are presented in table II illustrating the good results obtained. 


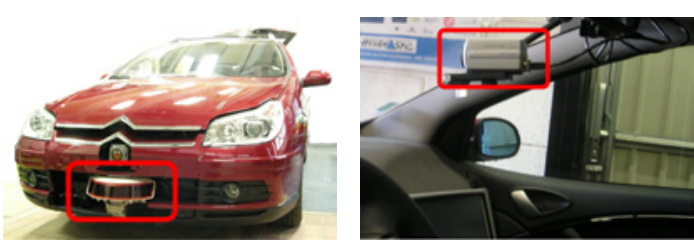

Fig. 6. Experimental platform: Lidar (left), Camera (right)

\begin{tabular}{|l||c||c|}
\hline \multicolumn{3}{|c|}{ Results of the Test using Real Data } \\
\hline Computation time & \multicolumn{2}{|c|}{460.912 seconds } \\
\hline Translation & $-0.1423 \mathrm{~m}$ & Confidence interval \\
\hline tx & $0.8398 \mathrm{~m}$ & $\pm 0.0628 \mathrm{~m}$ \\
\hline ty & $1.6994 \mathrm{~m}$ & $\pm 0.1043 \mathrm{~m}$ \\
\hline tz & & Confidence interval \\
\hline Rotation angles & $88.2011^{\circ}$ & $\pm 1.5871^{\circ}$ \\
\hline $\mathrm{Rx}$ & $-4.5321^{\circ}$ & $\pm 0.9449^{\circ}$ \\
\hline $\mathrm{Ry}$ & $90.7852^{\circ}$ & $\pm 1.5587^{\circ}$ \\
\hline $\mathrm{Rz}$ & \\
\hline
\end{tabular}

TABLE II

RESULTS ACHIEVED WITH REAL DATA

By using the rigid transformation, a projection of the multi-layer measurements into the scene image was made and illustrated in Figure7 and Figure 8. Some occlusions are revealed in Figure 8 which is coherent with respect to the observed scene.

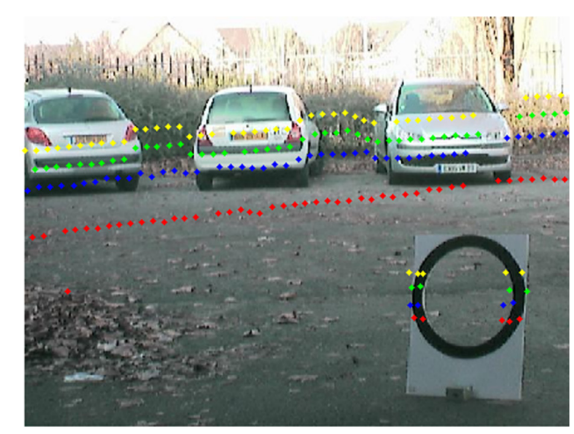

Fig. 7. Projection image of lidar data

\section{CONCLUSION}

A new extrinsic calibration method for a common sensor configuration in vehicle applications has been proposed. By using a circle-based calibration target, extrinsic calibration and intrinsic camera calibration can be effectuated simultaneously. The results obtained in real data tests illustrate an appropriate and accurate projection of the lidar data. The estimation of the confidence intervals in the calibration method allows taking into account the error propagation in data sensor fusion applications. The integration of a less-constrained camera model can improve the calibration method. It constitutes a perspective of this research.

\section{REFERENCES}

[1] R. Dupont, R. Keriven and P. Fuchs, "An improved calibration technique for coupled single-row telemeter and CCD," $3 D$ Digital Imaging and Modeling (3DIM); 2005

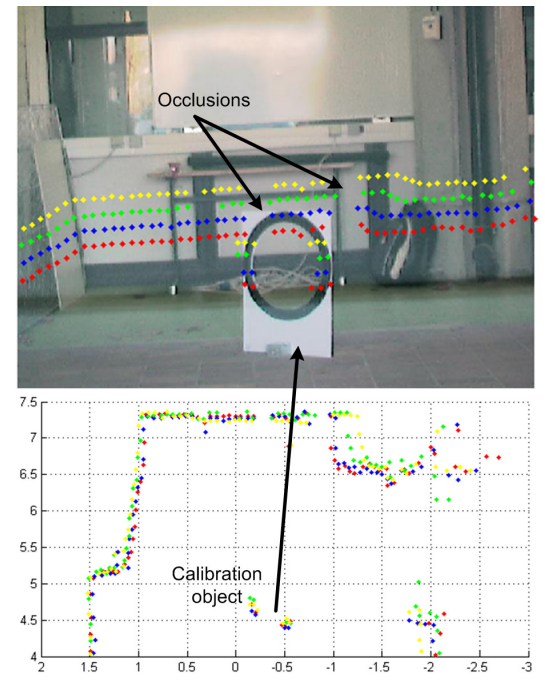

Fig. 8. Projection image of lidar data and (XY) plane view of 3-D lidar data

[2] Q. Zhang and R. Pless, "Extrinsic Calibration of a Camera and Laser Range Finder," In Proc. International Conference on Intelligent Robots and Systems; 2004.

[3] D. Scaramuzza, A. Harati and R. Siegwart, "Extrinsic Self Calibration of a Camera and a 3D Laser Range Finder from Natural Scenes," International Conference on Intelligent Robots and Systems.; 2007.

[4] A. Lorusso,D. Eggert and R. Fisher, "A comparison of four algorithms for estimating 3-d rigid transformations," British Machine Vision Conference; Vol. 1, pp. 237-246; 1995.

[5] C.M. Shakarji, "Least-squares fitting algorithms of the NIST algorithm testing system," Journal of Research of the National Institute of Standards and Technology, vol.103, pp. 633-641; 1998.

[6] D.W. Marquardt, "An algorithm for least-squares estimation of nonlinear parameters," Journal of the Society for Industrial and Applied Mathematics Vol. 11, pp. 431-441; 1963.

[7] P. Gurdjos, J. Kim and I. Kweon, "Geometric and Algebraic Constraints of Projected Concentric Circles and Their Applications to Camera Calibration," IEEE Trans. Pattern Anal. Mach. Intell.,Vol. 27, No. 4, pp. 637-642; 2005.

[8] V. Frémont and R. Chellali, "Direct Camera Calibration Using Two Concentric Circles from a Single View," Proc. Int'l Conf. Artificial Reality and Telexistence, pp. 93-98; 2002.

[9] K. Kanatani and N. Ohta, "Automatic Detection Of Circular Objects By Ellipse Growing," International Journal of Image and Graphics, Vol. 4, pp. 35-50; 2004.

[10] R. Hartley and A. Zisserman, "Multiple view geometry in computer vision," Cambrige University Press; 2003.

[11] R. Y. Tsai, "A versatile camera calibration technique for high accuracy 3D machine vision metrology using off-the-shelf TV cameras and lenses," IEEE J. Robotics Automat.,Vol. RA-3, No. 4, pp. 323-344; 1987.

[12] Y. Xie and Q. Ji, "A New Efficient Ellipse Detection Method," International Conference on Pattern Recognition,Vol. 2, pp. 957-960; 2002.

[13] W. Gander, G. Golub and R. Strebel, "Least-Squares Fitting of Circles and Ellipses," Technical Report of Computer Science Department, ETH Zürich; 1994.

[14] K. Arun, T. Huang and S. Blostein, "Least-squares Fitting of Two 3-D Point sets," IEEE Trans. Pattern Anal. Mach. Intell.,Vol. 9, No. 5, pp. 698-700; 1987.

[15] G. H. Golub and C. Van Loan, "Matrix Computations," The Johns Hopkins University Press. Third Edition; 1996.

[16] C. V. Stewart, "Robust Parameter Estimation in Computer Vision," Society for Industrial and Aplied Mathematics, Vol. 41, pp. 513-537; 1999.

[17] R. Lupton, "Statistics in Theory and Practice," Princeton University Press; 1993.

[18] P. Besl and N. McKay, "A Method for registration of 3-D Shapes," Trans. Pattern Analysis and Machine Intelligence, Vol. 14, No. 2; 1992. 